\title{
Study of Pc1 pearl structures observed at multi-point ground stations in Russia, Japan, and Canada
}

\author{
Chae-Woo Jun ${ }^{1 *}$, Kazuo Shiokawa ${ }^{1}$, Martin Connors ${ }^{2}$, lan Schofield ${ }^{2}$, Igor Poddelsky ${ }^{3}$ and Boris Shevtsov ${ }^{3}$
}

\begin{abstract}
We investigate possible generation mechanisms of Pc1 pearl structures using multi-point induction magnetometers in Athabasca in Canada, Magadan in Russia, and Moshiri in Japan. We selected two Pc1 pulsations that were simultaneously observed at the three stations and applied a polarization analysis. In case 1, on 8 April 2010, Pc1 pearl structures were slightly different in some time intervals at different stations, and their polarization angles varied depending on the frequencies at the three stations. Case 2, on 11 April 2010, showed Pc1 pearl structures that were similar at different stations, and their polarization angle was independent of frequency at all three stations. In order to understand these differences, we performed two simple model calculations of Pc1 pearl structures under different conditions. The first model assumes that Pc1 waves propagated from a latitudinally extended source with different frequencies at different latitudes to the observation points, representing beating of these waves in the ionosphere. The second model considers Pc1 waves for which different frequencies are mixed at a point source to cause the beating at the source point, indicating that the Pc1 pearl structures are generated in the magnetosphere. The first model shows slightly different waveforms at different stations. In contrast, the second model shows identical waveforms at different stations. From these results, we conclude that, in case 1, Pc1 pearl structures were caused by beating in the ionosphere. On the other hand, in case 2, they were the result of magnetospheric effects. We suggest that beating processes in the ionosphere could be one of the generation mechanisms of Pc1 pearl structures.
\end{abstract}

Keywords: Pc1 pulsations; Pearl structures; Multi-point ground-based observation

\section{Background}

Electromagnetic ion cyclotron (EMIC) waves are known to be generated in the equatorial region of the magnetosphere at $\mathrm{L}$ of approximately 4 to 8 (Anderson et al. 1992) due to ion cyclotron instability of energetic resonant ions with temperature anisotropy. These waves propagate along the geomagnetic field lines to the ionosphere, where they interact with the ionospheric plasma, generating compressional-mode waves. EMIC waves can generate isolated proton auroras via wave-particle interactions at subauroral latitudes (e.g., Sakaguchi et al. (2008); Nomura et al. (2012)). These ionospheric waves are observed as Pc1 geomagnetic pulsations in the frequency range of 0.2 to $5.0 \mathrm{~Hz}$. The characteristics of Pc1 pulsations in dynamic

\footnotetext{
*Correspondence: chae-woo@stelab.nagoya-u.ac.jp

1 Solar-Terrestrial Environment Laboratory, Nagoya University, Furo-cho, Chikusa-ku, Nagoya 464-8601, Japan

Full list of author information is available at the end of the article
}

spectra have been classified by Fukunishi et al. (1981). Pc1 pulsations can be trapped in the duct of the ionospheric F layer and propagate horizontally, from high to low latitudes, over long distances of several thousand kilometers (e.g., Manchester (1966); Tepley and Landshoff (1966); Campbell (1967); Kuwashima et al. (1981); Kawamura et al. (1981); Kim et al. (2011); Waters et al. (2013)).

Pc1 pearl structures are amplitude modulations of Pc1 waves, which show a quasi-periodic intensification of amplitude with a repetition period of several tens of seconds (Troitskaya and Gul'Elmi 1967). Pc1 pearl structures are the most common form of Pc1 pulsations. They have been studied for many years using groundbased and satellite observations, in order to understand the generation mechanisms of Pc1 pearl structures (e.g., Perraut (1982); Erlandson et al. (1990); Guglielmi et al. (1996); Mursula (2007); Rasinkangas and Mursula (1998); Mursula et al. (1999); Mursula (2007); Usanova et al.

\section{黑 Springer}

(c) 2014 Jun et al.: licensee Springer. This is an Open Access article distributed under the terms of the Creative Commons Attribution License (http://creativecommons.org/licenses/by/4.0), which permits unrestricted use, distribution, and reproduction in any medium, provided the original work is properly credited. 
(2008); Nomura et al. (2011)). However, the generation mechanisms of Pc1 pearl structures have yet to be clearly identified. For more than 50 years, the bouncing wave packets (BWP) model has been believed to be a possible generation mechanism of Pc1 pearl structures (e.g., Jacobs and Watanabe (1964); Obayashi (1965)). This model suggests that Pc1 pearl structures are caused by the bouncing of Pc1 waves along the geomagnetic field lines, between the northern and southern hemispheres. Mursula et al. (1999) found that there was a strong negative correlation between the repetition period of Pc1 pearl structures and the frequency of Pc1 pulsations. They implied that the L-shell dependence of various Pc1 frequencies, and the repetition period of Pc1 pearl structures may be interpreted by the BWP model. Nonetheless, recent studies have begun to consider whether or not the BWP model is the best mechanism to explain Pc1 pearl structures. For example, the fact that the repetition period of Pc1 pearl structures observed on the ground is shorter than that expected from the BWP model (e.g., Nomura et al. (2011); Usanova et al. (2008)) suggests that this model might not be entirely valid. Another possible generation mechanism of Pc1 pearl structures in the magnetosphere is the modulation of EMIC waves by long-period ultra-low frequency (ULF) waves, such as Pc4-5 pulsations (e.g., Mursula et al. (1997); Rasinkangas and Mursula (1998); Mursula et al. (2001); Mursula (2007)). Mursula et al. (1997) found a long Pc1 pulsation event on 11 April 1986 observed by the Finnish magnetometer network and the Viking satellite. They suggested that the pearl structures are modulated Pc1 waves propagating within or at the plasmapause, and that hydromagnetic chorus events consist of similar wave packets propagating outside the plasmasphere. Moreover, Rasinkangas and Mursula (1998) found that the EMIC waves observed by the Viking satellite in the inner magnetosphere were modulated by magnetospheric Pc3 pulsations. Hence, they proposed that the modulation of EMIC waves by long-period ULF waves could be an alternative to the BWP model. Other studies have also begun to consider ionospheric effects as a possible generation mechanism for Pc1 pearl structures. Pope (1964) and Nomura et al. (2011) suggested that beating in the ionosphere could create Pc1 pearl structures. Pope (1964) suggested that Pc1 waves with different frequencies are combined during their propagation through the ionospheric duct. Nomura et al. (2011) found that approximately $70 \%$ of the Pc1 pulsation events observed at low-latitude ground stations have a frequency dependence on the polarization angle. They inferred that these Pc1 events, coming from a spatially distributed ionospheric source, can create pearl structures by the beating of Pc1 waves with slightly different frequencies in the ionosphere. However, previous studies have not investigated what type of generation mechanisms, either in the magnetosphere or in the ionosphere, contribute more to the formation of Pc1 pearl structures.

In this study, we report two Pc1 pulsation events simultaneously observed at longitudinally and latitudinally separated ground stations. In order to consider Pc1 pulsations coming from the same source region, observed simultaneously at three stations, we used a coherence analysis of their waveforms. Moreover, we investigated the similarity of Pc1 pearl structures between two stations using a cross correlation analysis of the upper envelopes of Pc1 pearl structures. Details of the analyses are presented in the 'Observations' subsection. We found two Pc1 pulsations simultaneously observed at these three stations. In case 1 , the Pc1 pearl structures are slightly different at different stations, whereas in case 2, they are similar at different stations. According to our model calculations of Pc1 pearl structures, we found that a spatially distributed ionospheric source can create different waveforms at different stations. This research is the first case study comparing the similarity of Pc1 pearl structures at very distant ground stations. This research provides evidence suggesting that beating in the ionosphere is one of the possible generation mechanisms of Pc1 pearl structures.

\section{Methods \\ Observations}

We investigated Pc1 pearl structures using multipoint induction magnetometers deployed by the SolarTerrestrial Environment Laboratory, Nagoya University, at Athabasca (ATH) in Canada, Magadan (MGD) in Russia, and Moshiri (MOS) in Japan. Figure 1 shows the locations of these ground stations, giving their geographic latitudes and longitudes, as well as dipole geomagnetic latitudes, calculated using the IGRF-11 model with an epoch time of 2010. The distance between ATH and MGD is about 4,000 km, while MGD and MOS are separated by about $1,000 \mathrm{~km}$. In this study, we use the data giving the H, D, and $\mathrm{Z}$ geomagnetic field components with a sampling rate of $64 \mathrm{~Hz}$ and a GPS clock accuracy of about $1 \mu \mathrm{s}$. The magnetometer sensors have almost identical sensitivities and provide phase differences for $\mathrm{H}, \mathrm{D}$, and $\mathrm{Z}$ components in the Pc1 frequency range $(0.2$ to $5.0 \mathrm{~Hz})$. The magnetometer data, originally in volts, were converted to nT units by considering the frequency-dependent amplitude of the system. The sensor sensitivity was measured by a calibration coil with a length of $2 \mathrm{~m}$. Additional details of these induction magnetometers are given by Shiokawa et al. (2010).

\section{Data analysis and event selection}

The power spectrum density (PSD) of the two horizontal magnetic field components, $\mathrm{H}$ and $\mathrm{D}$, measured by the induction magnetometers, was calculated by fast Fourier transform (FFT) every $30 \mathrm{~s}$. We used a time window of 


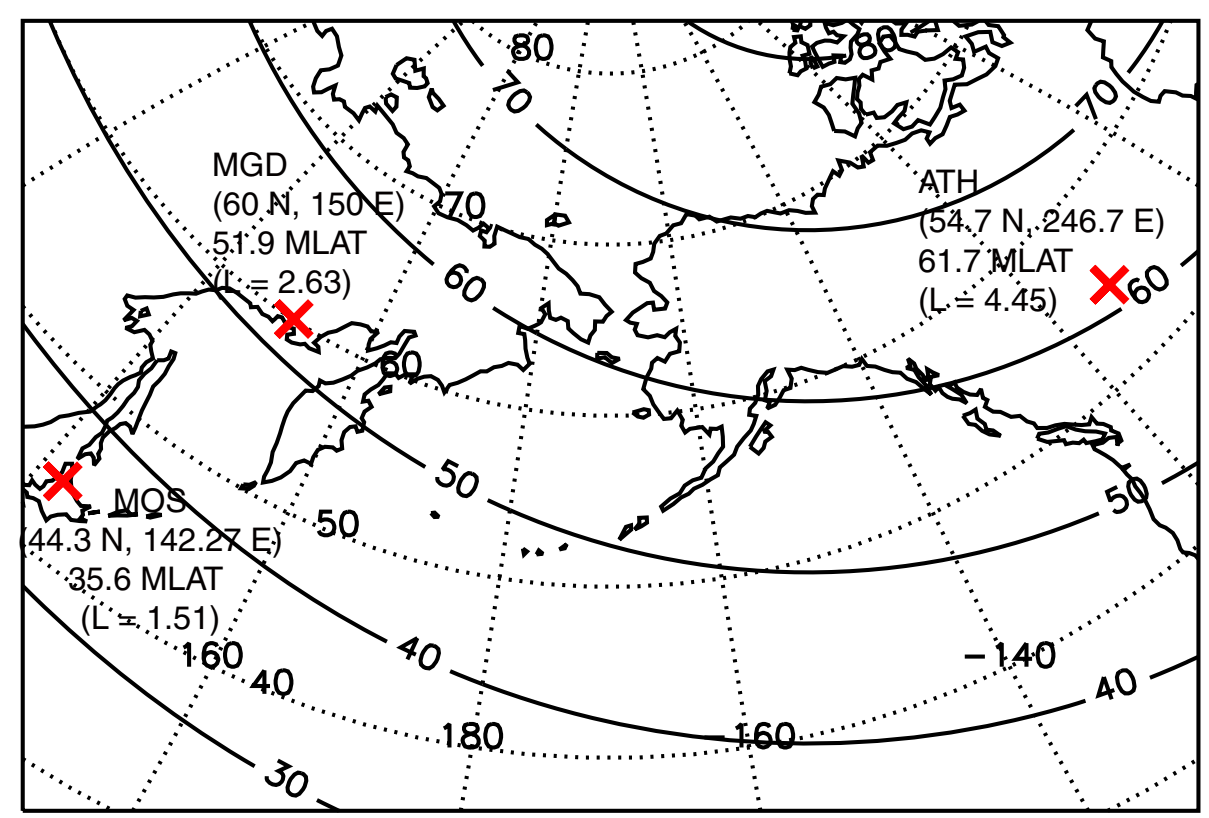

Figure 1 Location of the three induction magnetometer stations. These stations are located at Athabasca (ATH) in Canada, Magadan (MGD) in Russia, and Moshiri (MOS) in Japan. Solid lines indicate dipole magnetic latitudes calculated using the IGRF-11 model with an epoch time of 2010. Dashed lines indicate geographic coordinates.

$128 \mathrm{~s}$, with a frequency resolution of $0.0078 \mathrm{~Hz}$. In each window, the polarization angle is calculated using the relationship described in Fowler et al. (1967). The polarization angle is defined as the positive (negative) angle measured from the magnetic north westward (eastward). The coherence of the Pc1 waveforms helps us to determine whether or not the Pc1 pulsations come from the same source region in the frequency domain. If the coherence of Pc1 waveforms is close to one, with the same frequencies between two signals, the two signals are identical, indicating that these two signals are coming from the same source. On the other hand, if the coherence is equal to zero, the two signals have no correlation, indicating that these two signals are coming from two different sources.

From 1 January 2009 to 31 December 2011, we investigated three years of dynamic spectral data of geomagnetic pulsations obtained at ATH, MGD, and MOS. We considered four criteria in selecting Pc1 pulsations by visual inspection of the spectra: Pc1 pulsations are well-defined in the PSD and within the frequency range of 0.2 to 5.0 $\mathrm{Hz}$; the Pc1 pulsation power should be greater than $10^{-4}$ $\mathrm{nT}^{2} / \mathrm{Hz}$; the Pc1 frequency bandwidth should be wider than $0.2 \mathrm{~Hz}$; the Pc1 pulsation should be discernible for more than $20 \mathrm{~min}$.

Using these criteria, we selected 509 Pc1 events at ATH, 366 at MGD, and 518 at MOS. Then, we chose 28 Pc1 pulsations that were observed simultaneously at these three stations. We defined those events that have a coherence value greater than 0.5 as high coherence events.
The number of high coherence events observed between ATH and MGD was seven. Only two of those events were observed clearly at all three stations without contamination by other frequency bands of Pc1 pulsations. We selected two Pc1 pulsations that show similar shapes in the dynamic spectra at all three stations: case 1 on 8 April 2010, and case 2 on 11 April 2010.

\section{Results}

\section{Case 1 - 8 April 2010}

Figure 2 shows the dynamic spectra of $\mathrm{H}$ and D components of the magnetic field variations, polarization angle, coherence between different stations, and crosscorrelation of Pc1 amplitude envelopes (red lines in Figure 3), for a clear Pc1 geomagnetic pulsation event observed simultaneously at ATH, MGD, and MOS at 10:00 to 12:00 UT on 8 April 2010. We chose the crosscorrelation between the ATH H component and the MGD D component, since they show the highest correlation between ATH and MGD. During this event, geomagnetic activity was slightly elevated ( $K_{p}=1$ to 2$)$, with AE indices of approximately 300 to $500 \mathrm{nT}$. The local time at ATH was 00:00 to 02:00 LT, and the local time at MGD and MOS was 06:00 to 08:00 LT. In Figure 2a,b,c,d,e,f, Pc1 pulsations can be clearly identified at the three stations in the frequency range of 0.4 to $1.2 \mathrm{~Hz}$. The intensity of PSD at MOS is weaker than at the other two stations, probably because of attenuation due to ionospheric duct propagation to lower latitudes (Kawamura et al. 1981). We can 


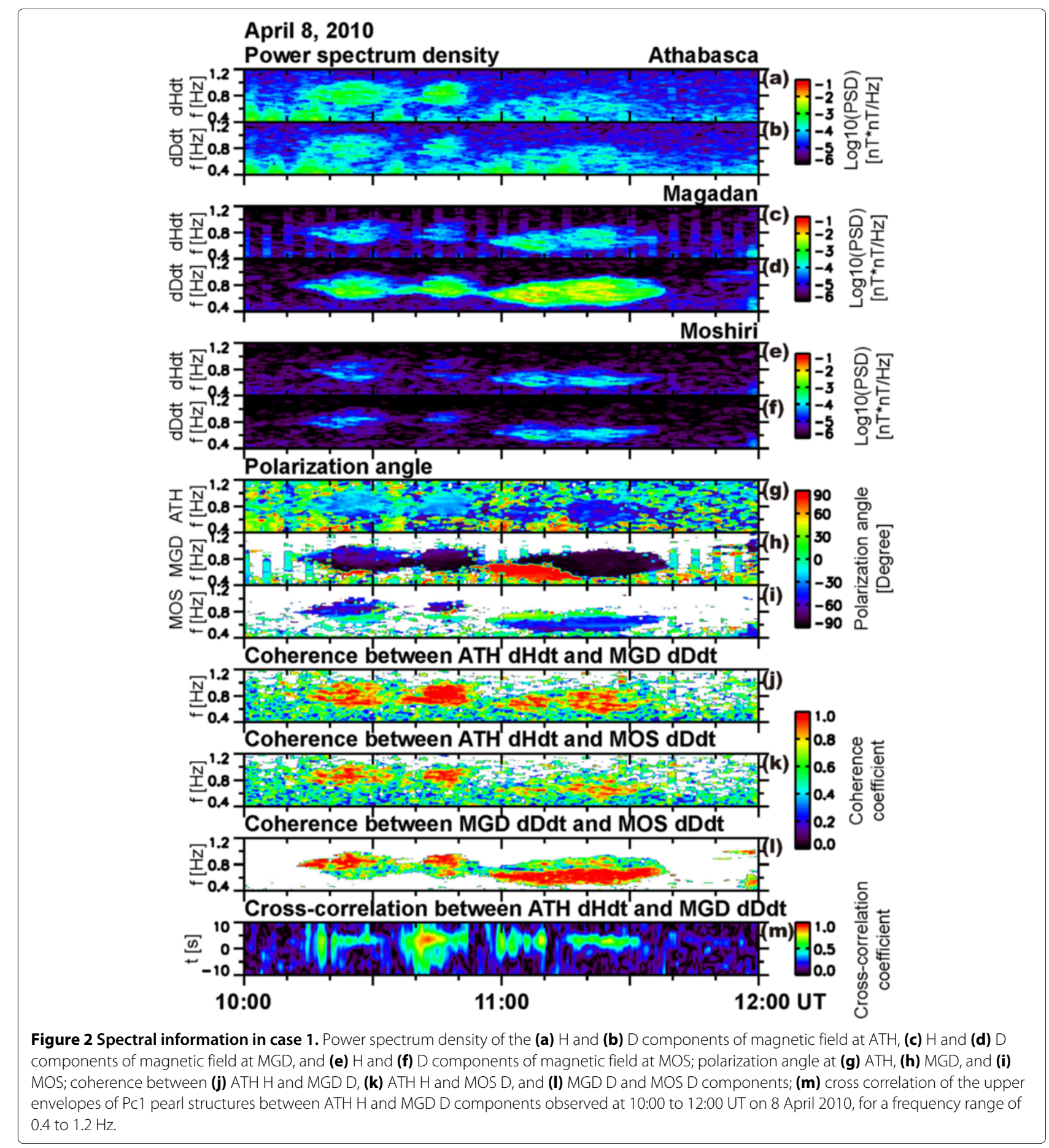

see that the Pc1 pulsations are observed during three separated time intervals: $10: 12$ to $10: 33,10: 41$ to $10: 53$, and 10:55 to 11:49 UT at all stations. At ATH, the first and second Pc1 bursts can be clearly seen in Figure 2a,b, but the third Pc1 burst is much weaker than the others. On the other hand, Pc1 pulsations are clearly identifiable at MGD and MOS for all three intervals.
The polarization angle at $\mathrm{ATH}, \mathrm{MGD}$, and MOS is shown in Figure 2g,h,i. For ATH (Figure 2g), it increased from approximately $-50^{\circ}$ (dark blue) to $-20^{\circ}$ (light blue) for 0.6 to $1 \mathrm{~Hz}$. At MGD (Figure 2h), the polarization angle varies from approximately $-90^{\circ}$ (black) to $-50^{\circ}$ (dark blue) in the same frequency range as ATH. The frequency dependence at MOS (Figure 2i) shows a decrease of the 
a April 8, 2010

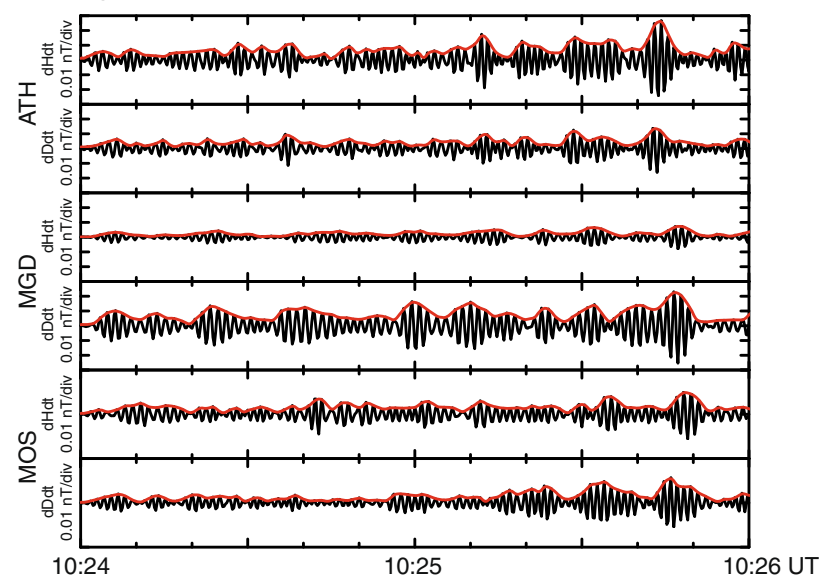

b

b April 8, 2010

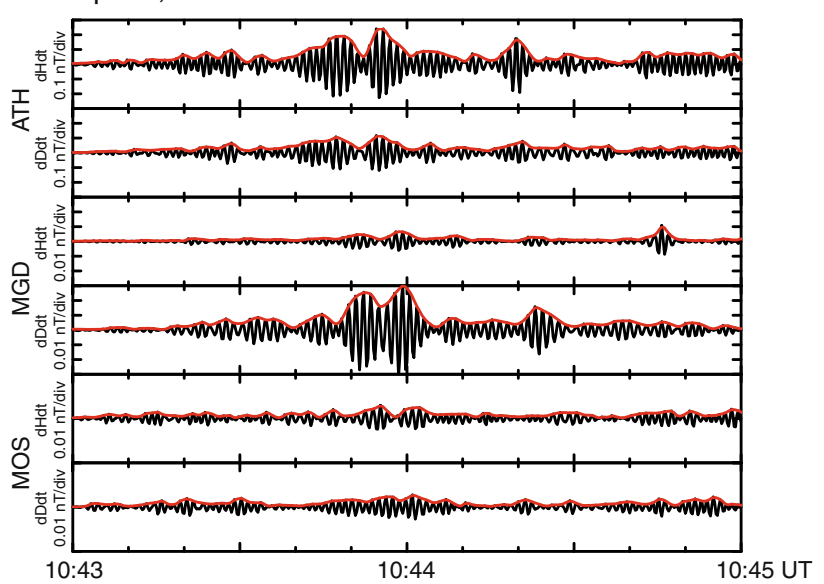

Figure 3 Time series analysis in case 1. (Top to bottom) band-pass filtered ( 0.5 to $1.2 \mathrm{~Hz}$ ) Pc1 waveform of the magnetic field of the $\mathrm{H}$ and $\mathrm{D}$ components observed at ATH, MGD, and MOS at (a) 10:24 to 10:26 and (b) 10:43 to 10:45 UT on 8 April 2010. Red solid lines indicate upper envelope of Pc1 pearl structures.

polarization angle from approximately $30^{\circ}$ (green) to $-50^{\circ}$ (dark blue). According to Nomura et al. (2011), this polarization angle dependence on frequency indicates that the Pc1 pulsation observed at the three stations at 10:00 to 12:00 UT has a spatially distributed ionospheric source at high latitudes.

In order to distinguish whether the Pc1 pulsations propagated from the same ionospheric source, we show the coherence of Pc1 waveforms between each pair of stations in Figure 2j,k,l. High coherence of Pc1 waveforms was observed between two stations, indicating that the Pc1 pulsations observed at the three different stations were propagated from the same origin in the ionosphere.

Figure 3a,b shows the waveforms of the $\mathrm{H}$ and $\mathrm{D}$ components of Pc1 pulsations at ATH, MGD, and MOS on 8 April 2010 at 10:24 to 10:26 and 10:43 to 10:45 UT, respectively. In order to remove noise from other frequencies, we show the time series of Pc1 pulsations obtained using a band-pass filter in the frequency range of 0.5 to $1.2 \mathrm{~Hz}$. The amplitude modulation of the pulsations varies with a repetition period of approximately $10 \mathrm{~s}$ in both time intervals. The time difference between ATH and MGD (MGD and MOS) is approximately $4 \mathrm{~s}(0 \mathrm{~s})$ and was confirmed by lag correlation studies (see below). The repetition periods of pearl structure changes in time during this event at all stations, and the ones observed at ATH, MGD, and MOS are generally similar but differ in their details.

Figure 4 shows the PSD of the $\mathrm{H}$ and $\mathrm{D}$ components of magnetic field variations at ATH and MGD, respectively, on 8 April 2010 and the coherence of Pc1 waveforms between these two components at 10:24:00 to 10:26:08 (Figure 4a,b,c) and 10:43:00 to 10:45:08 UT (Figure 4c,d,e) with a resolution of $0.0078 \mathrm{~Hz}$. The time intervals of Figure 4a,b,c,d,e correspond to those shown in Figure 3a,b. In Figure 4a,b and Figure 4d,e, we can see a continuous high-PSD band at frequencies of 0.6 to 1.0 and 0.7 to $1.0 \mathrm{~Hz}$, respectively. The coherence between the $\mathrm{H}$ component at ATH and the D component at MGD is close to one in the latter frequency range.

We investigated the cross-correlation between the $\mathrm{H}$ component at ATH and the D component at MGD using the upper envelope of Pc1 pearl structures in the time domain. We used only these components because they have the highest PSD intensity compared with the other components and the background intensity. Figure 5a,b shows the cross-correlation between the $\mathrm{H}$ component at ATH and D component at MGD obtained for the upper envelope of the Pc1 pearl structure at 10:24:00 to 10:26:08 and 10:43:00 to 10:45:08 UT, respectively, on 8 April 2010. In Figure 5a, we can see that the correlation is greater than 0.5 with a time difference of approximately $3 \mathrm{~s}$. On the other hand, in Figure 5b, the cross-correlation between ATH and MGD is close to 0.9, indicating that the upper envelope of the Pc1 pearl structures is generally similar, with a time difference of approximately $3 \mathrm{~s}$. In Figure $2 \mathrm{~m}$, the cross-correlation between the $\mathrm{H}$ component at ATH and the D component at MGD is greater than 0.5 throughout this event. Especially, for the second of the Pc1 bursts, the coherence and correlation are both extremely high $(r>0.8)$. For the first and third Pc1 burst time intervals, however, the correlation decreases to 0.5 , even if the coherence of the Pc1 waveforms is clearly close to one.

\section{Case 2 - 11 April 2010}

Figure 6 shows the dynamic spectra of the $H$ and D components of magnetic field variations, polarization 
April 8, 2010
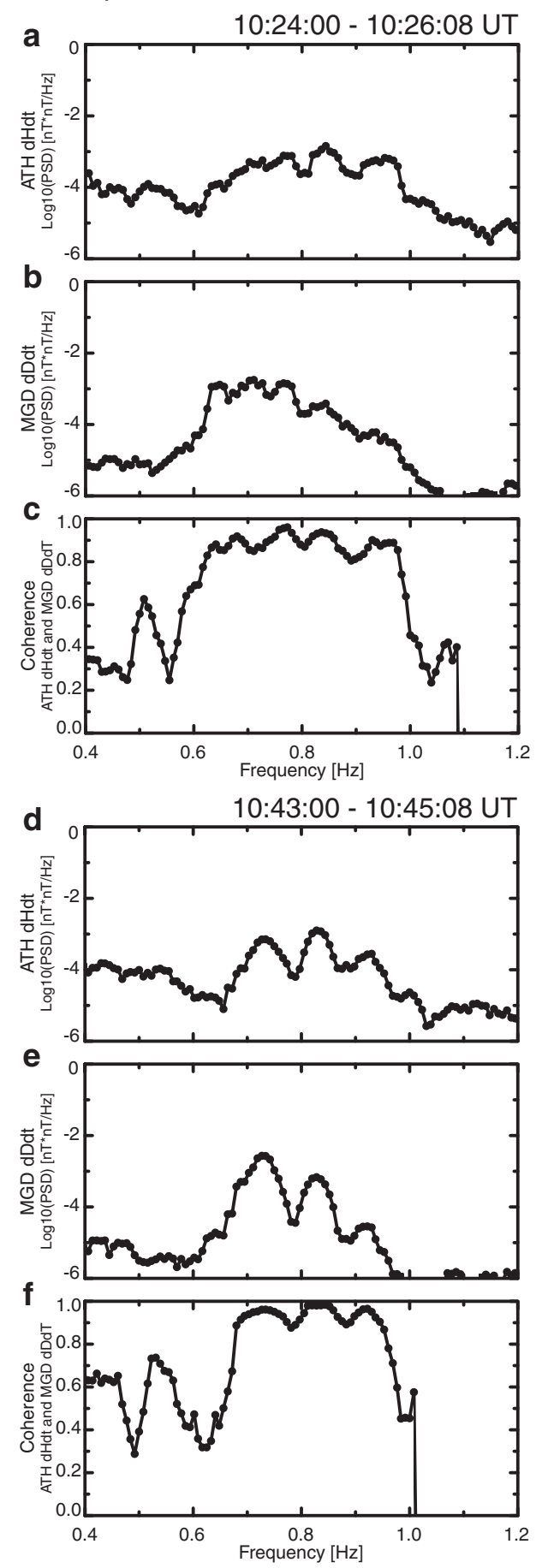

Figure 4 The PSD and the coherence of Pc1 waveforms of the $\mathrm{H}$ and $\mathrm{D}$ components in case 1. Upper three panels show the power spectrum density of (a) $\mathrm{H}$ component of the magnetic field at ATH and (b) D component of the magnetic field at MGD, as well as (c) coherence of Pc1 waveforms between the $\mathrm{H}$ component at ATH and D component at MGD, observed at 10:24:00 to 10:26:08 UT on 8 Apri 2010, for a frequency range of 0.4 to $1.2 \mathrm{~Hz}$. Lower three panels (d-f) show the same quantities observed at 10:43:00 to 10:45:08 UT on 8 April 2010. angle, coherence between different stations, and crosscorrelation of Pc1 amplitude envelopes (red lines in Figure 7) between the ATH H component and the MGD D component, for a clear Pc1 geomagnetic pulsation event observed simultaneously at ATH, MGD, and MOS at 11:00 to 13:00 UT on 11 April 2010. Geomagnetic activity was relatively high during this event, with $K_{p}=3$ to 4. The average AE index during the 11:00 to 13:00 UT interval was approximately $104 \mathrm{nT}$. The local time at ATH was 01:00 to 03:00 LT, and 07:00 to 09:00 LT at MGD and MOS. In Figure 6a,b,c,d, Pc1 pulsations were clearly identified at ATH and MGD in the frequency range of 0.2 to $0.8 \mathrm{~Hz}$. However, the Pc1 pulsations observed at MOS were much weaker than those seen at the other two stations (Figure 6e,f). At 11:30 to 12:20 UT at ATH, we can see three different frequency bands of Pc1 pulsations at 0.38 to $0.48,0.5$ to 0.6 , and 0.61 to $0.73 \mathrm{~Hz}$. At MGD, the two different frequency bands of Pc1 pulsations at 0.4 to 0.47 and 0.52 to $0.6 \mathrm{~Hz}$ can be seen in the $\mathrm{D}$ component of magnetic field variation (Figure $6 \mathrm{~d}$ ).

The polarization angles at ATH, MGD, and MOS are shown in Figures 6g-6i. From $0.3 \mathrm{~Hz}$ to $0.7 \mathrm{~Hz}$, the polarization angle at ATH (Figure 6g) is almost constant, with values around $0^{\circ}$ (light green). In Figure $6 \mathrm{~h}$, the polarization angle at MGD barely varies, and remains near $-60^{\circ}$ (dark blue) in the frequency range of 0.3 to $0.6 \mathrm{~Hz}$. The frequency dependence at MOS, as seen in Figure 6i, is not clear because the intensity of the Pc1 pulsations is very weak. The constant polarization angle observed at ATH and MGD, independent of frequency, suggests that Pc1 pulsations have a localized ionospheric source at high latitudes (Nomura et al. 2011).

In order to distinguish whether Pc1 pulsations propagated from the same ionospheric source, we show the coherence of Pc1 waveforms between the different stations in Figure 6j,k,l. The coherence between two stations is close to one for all frequencies, even if the Pc1 pulsation at MOS was weak for frequencies near $0.5 \mathrm{~Hz}$. This indicates that the Pc1 pulsations observed at the three different stations propagated from the same origin.

Figure 7a,b shows the waveforms of the $\mathrm{H}$ and $\mathrm{D}$ components of Pc1 pulsations observed at ATH, MGD, and MOS on 11 April 2010 at 11:57 to 11:59 and 12:02 to 12:04 UT, respectively. We show the time series of Pc1 pulsations obtained using a band-pass filter from 0.3 to $0.7 \mathrm{~Hz}$ in order to remove noise from other frequencies. Even if the amplitude modulation of Pc1 pulsations at MOS was weak in both time intervals, we can clearly see Pc1 pearl structures in all three stations. The repetition periods of Pc1 pearl structures vary from 10 to $40 \mathrm{~s}$. We also note that there is a time difference of a few seconds in the pearl structures between ATH and MGD. The Pc1 pearl structures observed at ATH and MGD are similar in the time series of magnetic field variation, even though these 
April 8, 2010

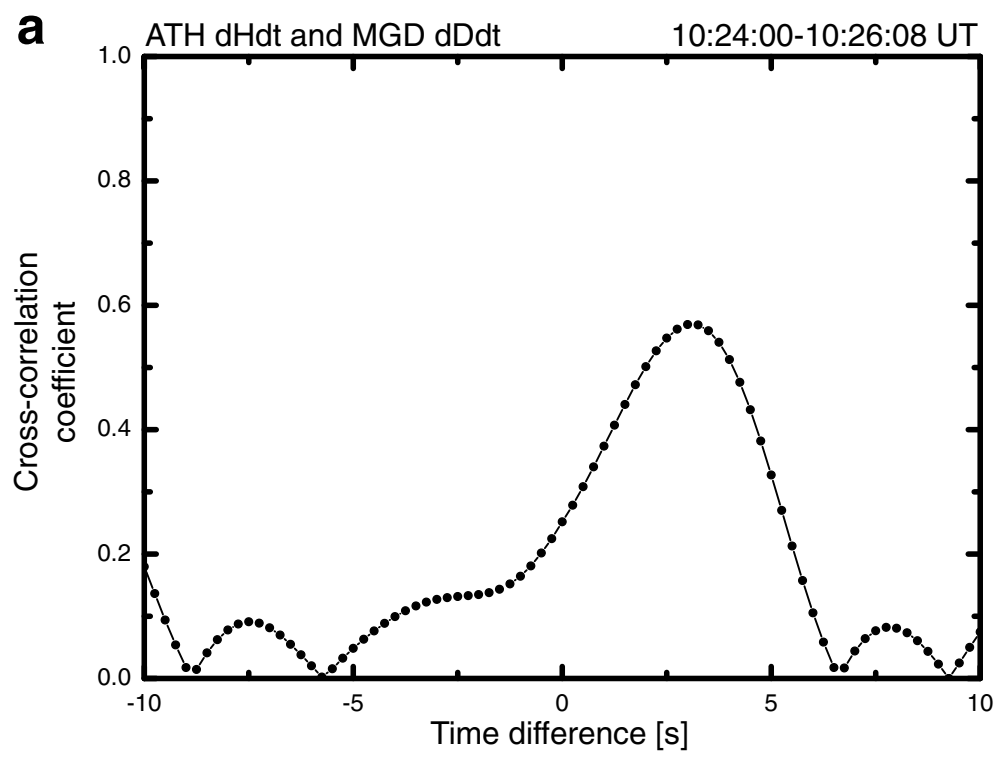

April 8, 2010

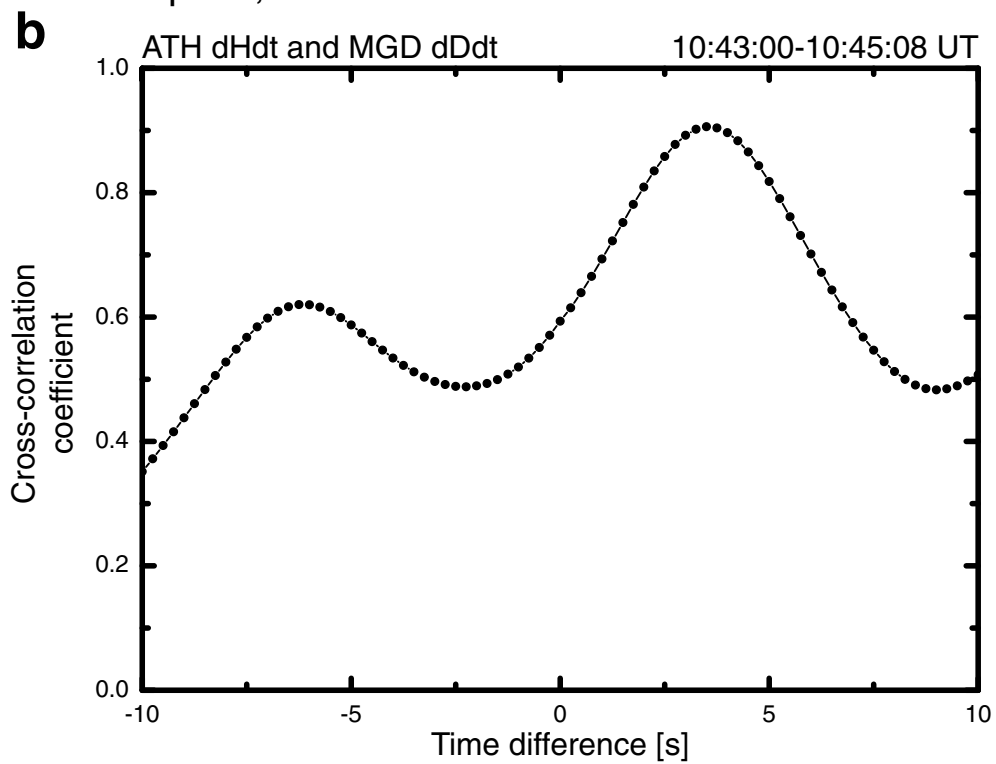

Figure 5 The cross-correlation analysis in case 1. Cross-correlation between $\mathrm{H}$ component at ATH and D component at MGD, obtained using upper envelope of Pc1 pearl structures at (a) 10:24:00 to 10:26:08 and (b) 10:43:00 to 10:45:08 UT on 8 April 2010.

two stations are separated by a distance of approximately $4,000 \mathrm{~km}$.

Figure 8 shows the PSD of the $\mathrm{H}$ and $\mathrm{D}$ component magnetic field variations at ATH and MGD, respectively, and the coherence between these two components at 11:57:00 to 11:59:08 (Figure 8a,b,c) and 12:02:00 to12:04:08 UT (Figure 8c,d,e) on 11 April 2010, with a frequency resolution of $0.0078 \mathrm{~Hz}$. The time intervals in Figure 8a,b correspond to those shown in Figure $7 \mathrm{a}, \mathrm{b}$, respectively. In Figure $8 \mathrm{a}, \mathrm{b}$ and Figure 8d,e, we can see a continuous
high-PSD band at frequencies of 0.4 to $0.6 \mathrm{~Hz}$ in both time intervals. The coherence between the $\mathrm{H}$ component at ATH and the D component at MGD is close to one in the frequency range of 0.4 to $0.6 \mathrm{~Hz}$.

Figure 9a,b shows the cross-correlation between the upper envelopes of ATH H and MGD D components of Pc1 pearl structure at 11:57:00 to 11:59:08 and 12:02:00 to 12:04:08 UT, respectively, on 11 April 2010, in the same format as Figure 5. In Figure 9a,b, we can see that the correlation is greater than 0.8 with a time difference of 


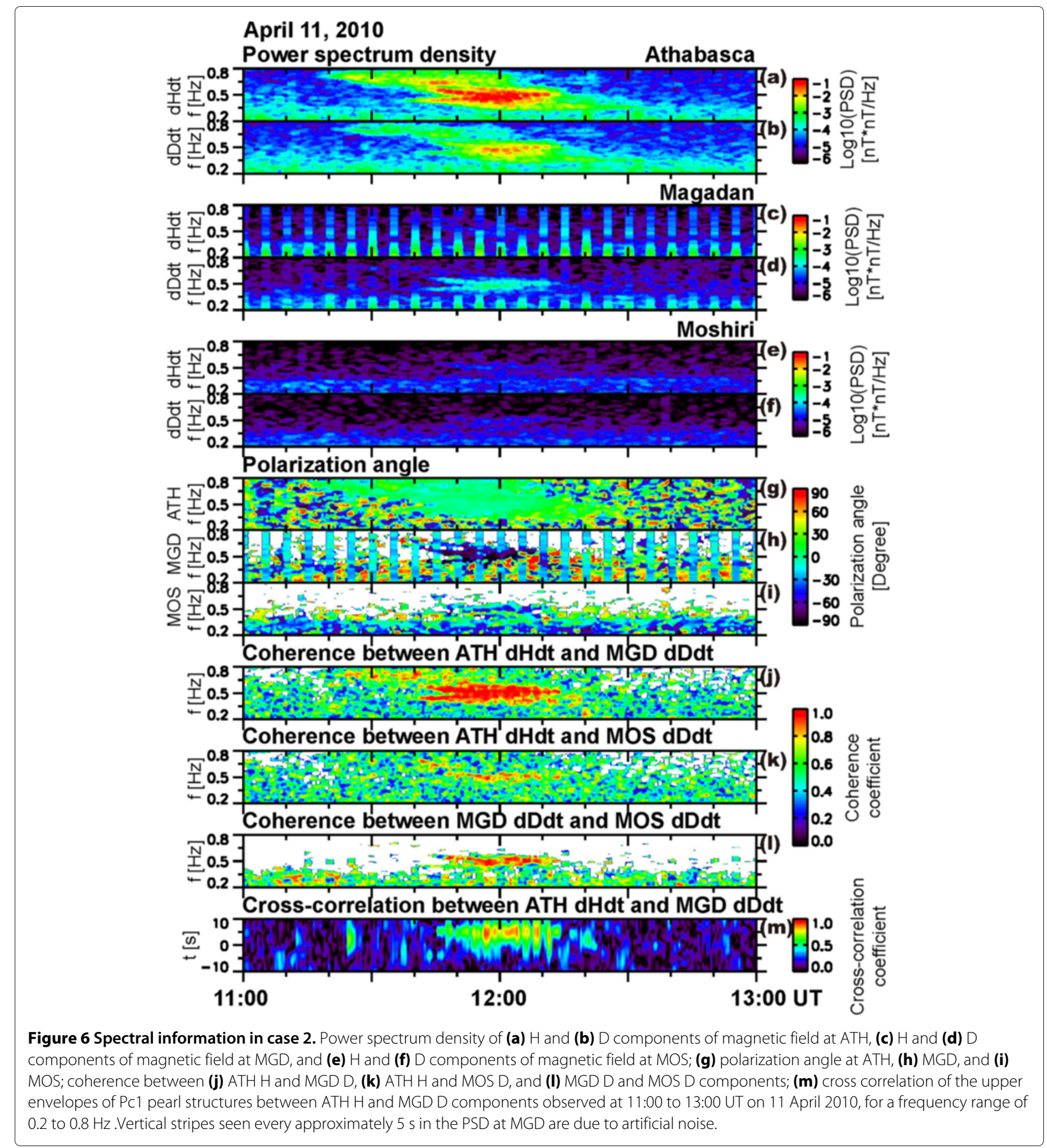

approximately $4 \mathrm{~s}$ during both time intervals. As shown in Figure 6m, we made this cross-correlation analysis of Pc1 envelopes for the whole time interval. The crosscorrelation of Pc1 envelopes between the $\mathrm{H}$ component at ATH and the D component at MGD is greater than 0.8 throughout this Pc1 event, which also has high coherence $(r>0.8)$.

\section{Discussion}

Comparing two case studies, we found that the Pc1 pearl structures observed at widely separated ground stations can be generally similar. However, case 1 shows that detailed pearl structures are different in some time intervals, even if the coherence of Pc1 waveforms between two different stations is close to one. The polarization angle 


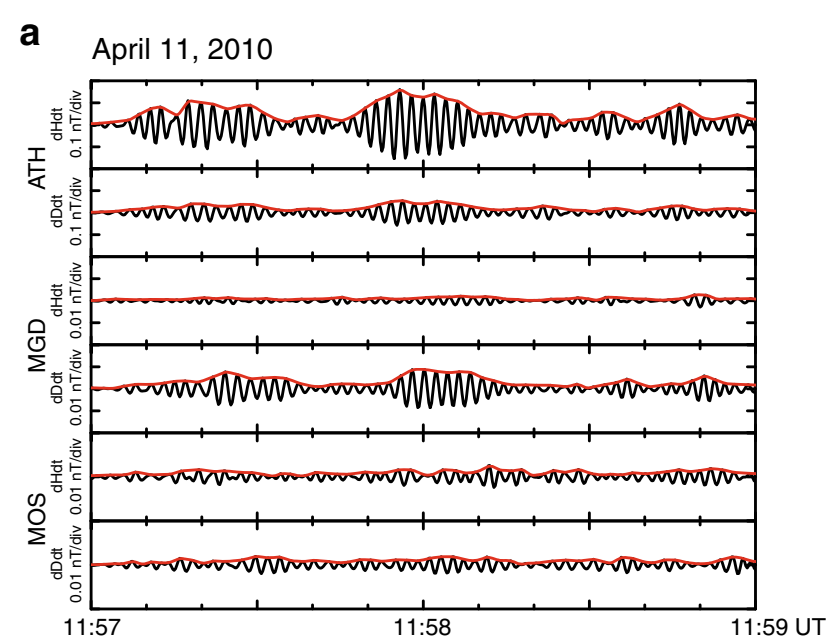

b

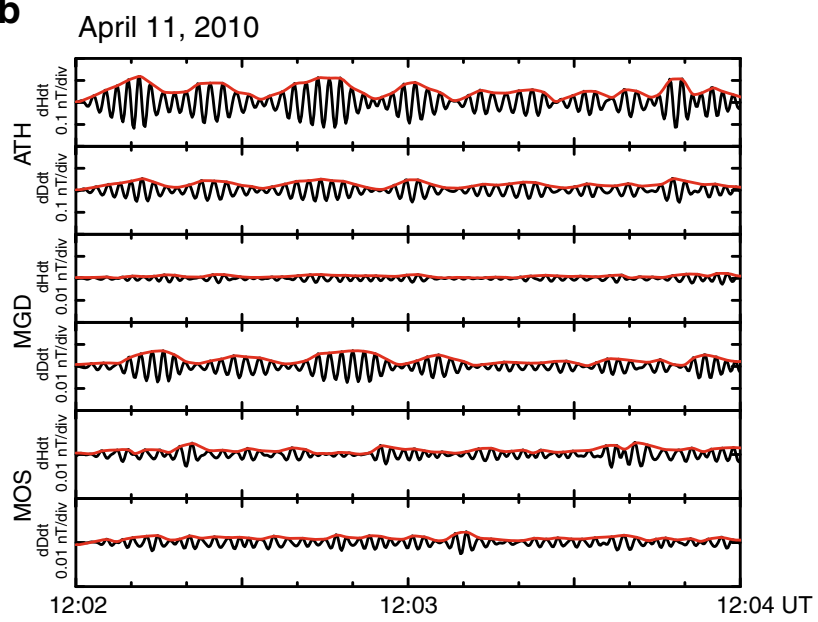

Figure 7 Time series analysis in case 2. (Top to bottom) band-pass filtered $(0.3$ to $0.7 \mathrm{~Hz}$ ) Pc1 waveforms of the magnetic field of $\mathrm{H}$ and $\mathrm{D}$ components observed at ATH, MGD, and MOS at (a) 11:57 to 11:59 and (b) 12:02 to 12:04 UT on 11 April 2010. Red solid lines indicate upper envelope of Pc1 pearl structures.

varied depending on frequency for case 1, suggesting a spatially distributed ionospheric source. On the other hand, case 2 shows that the coherence of Pc1 waveforms and cross-correlation of Pc1 envelopes can both be high $(r>0.8)$. In this second case, the polarization angle was almost constant for frequencies from 0.4 to $0.6 \mathrm{~Hz}$. Here, we discuss the mechanisms that may have contributed to these differences.

\section{Possible generation mechanisms of Pc1 pearl structures in the magnetosphere}

One of the possible generation mechanisms is based on the BWP model (e.g., Guglielmi et al. (1996); Mursula et al. (1999)). This model explains that Pc1 pearl structures are caused by bouncing of Pc1 waves along the geomagnetic field line between the northern and southern hemispheres. According to the BWP model, the repetition period of Pc1 pearl structures would be related to the length of the magnetic field line, as well as to the Alfven velocity in the magnetosphere. According to this model, the expected repetition period of Pc1 pearl structures is several tens of seconds, depending on the radial distance of the generation region of EMIC waves located near the magnetic equator. However, some studies have reconsidered the BWP model, because they found that the observations did not match the expected results from this model. For example, since the BWP model is based on comparison of ground and satellite data, Perraut (1982) found that the repetition period of Pc1 pearl structures seen on the ground station, did not clearly match the one observed in space. In addition, Erlandson et al. (1990) measured the Poynting flux of EMIC waves using the Viking satellite to investigate Pc1 pulsations near the plasmapause. They found that the energy flux of Pc1 pearl structures was mainly downward, along the magnetic field line. Moreover, Paulson et al. (2014) measured the average wave power over 0.6 to $0.8 \mathrm{~Hz}$ of Pc1 waves observed at the Hornsund station on the ground and the Van Allen probe in space. They found that both repetition periods of an average wave power were approximately $130 \mathrm{~s}$. They suggested that the similar repetition periods on the ground and in space contradict the BWP model, because if the BWP model is correct, the repetition period of average wave power in space would have to be half of that observed on the ground. In Figures 3 and 7, the repetition period of Pc1 pearl structures at three stations was approximately $10 \mathrm{~s}$, which is shorter than the expected repetition period from the BWP model. Mursula et al. (2001) and Mursula (2007) attempted to explain the Pc1 pearl structures as the result of modulation of EMIC waves by long-period ULF waves (such as Pc4 to 5 pulsations). The repetition periods of Pc1 pearl structures found in this study, approximately $10 \mathrm{~s}$ in case 1 , and approximately 10 to $40 \mathrm{~s}$ in case 2 , are shorter than the period of Pc4 to 5 pulsations. Such generation mechanisms of Pc1 pearl structures in the magnetosphere are not able to explain the different wave structures at different stations that we observed, even in the case of Pc1 pulsations that propagated from the same source. If Pc1 waves with different frequencies are mixed in the magnetosphere, these waves should have similar waveforms, even if they are detected at different stations. As we observed in case 1 , the detailed Pc1 pearl structures were slightly different at the three stations. In Figure $2 \mathrm{~m}$, the cross-correlation of Pc1 envelopes at ATH and MGD is less than 0.5 in the time interval of the first and third Pc1 
April 11, 2010
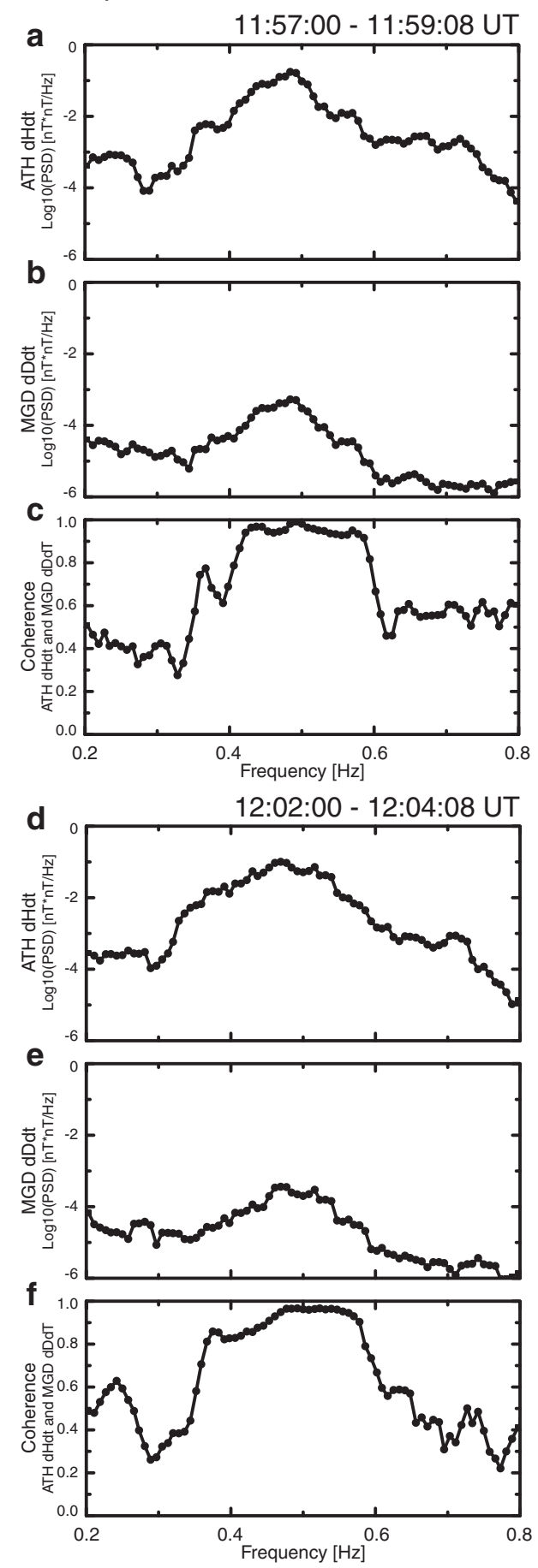

Figure 8 The PSD and the coherence of Pc1 waveforms of the $\mathrm{H}$ and D components in case 2 . Upper three panels show the power spectrum density of (a) $\mathrm{H}$ component of the magnetic field at ATH and (b) D component of the magnetic field at MGD, as well as (c) coherence of PC1 waveforms between $\mathrm{H}$ component at ATH and D component at MGD, observed at 11:57:00 to 11:59:08 UT on 11 April 2010, for a frequency range of 0.2 to $0.8 \mathrm{~Hz}$. Lower three panels (d-f) show the same quantities observed at 12:02:00 to 12:04:08 UT on 11 April 2010. bursts, although the coherence of Pc1 waveforms is close to one.

\section{Comparison of observations and model calculations of Pc1 pearl structures}

Some studies have considered that Pc1 pearl structures can be caused by beating in the ionosphere. This is the consequence of amplitude modulation of Pc1 waves caused by superposition of the waves at slightly different frequencies during their propagation through the ionospheric duct (Pope 1964). Nomura et al. (2011) found that some Pc1 events observed at low latitudes have a polarization angle that is frequency dependent. This indicates that these Pc1 pulsations have a spatially distributed source region in the ionosphere that can cause beating in the ionosphere to create Pc1 pearl structures. In case 1, Figure 2g,h,i shows that $\mathrm{Pc} 1$ pulsations at three different stations have a polarization angle that is dependent on frequency, and thus we can suggest that these waves have a spatially distributed source in the ionosphere. Figure $3 \mathrm{a}, \mathrm{b}$ shows that the Pc1 pearl structures varied with a repetition period of $10 \mathrm{~s}$, suggesting that these structures may also be caused by beating in the ionosphere. Moreover, their amplitude envelopes are slightly different at the three stations. On the other hand, in case 2, the polarization angle does not show any dependence on frequency (Figure 6g,h,i), indicating that these waves have a localized ionospheric source. Figure 7a,b shows that the Pc1 pearl structures at the three stations are similar, with a repetition period of approximately $10 \mathrm{~s}$.

To understand the different features of Pc1 pearl structures between the two case studies, we did two model calculations of Pc1 pearl structures under two different conditions, as shown in Figure 10. We generated simple sinusoidal waves in the frequency range of 0.6 to $1.0 \mathrm{~Hz}$, and assumed that they propagated through the ionospheric duct with an Alfven velocity of $500 \mathrm{~km} / \mathrm{s}$, as estimated by Fraser (1975). We took into account the time difference and polarization angle variation due to the relative location of these wave sources and the observation points. We did not consider the mode conversion effect and polarization sense of Pc1 waves during their propagation through the ionospheric duct. During the duct propagation, we consider an attenuation effect that causes the amplitude of Pc1 waves to decrease by $25 \%$ per 1,000 $\mathrm{km}$ distance from the source region to each station. Subsequently, we checked the pearl structures under two different conditions. First, model 1 assumes that the Pc1 waves are generated at a north-south extended source region (orange line in Figure 10a) with frequencies from 0.6 (high latitude) to $1.0 \mathrm{~Hz}$ (low latitude) at different latitudes. This would correspond to a pearl structure caused by beating during duct propagation in the ionosphere, from their source points to the observation points. Second, model 2 


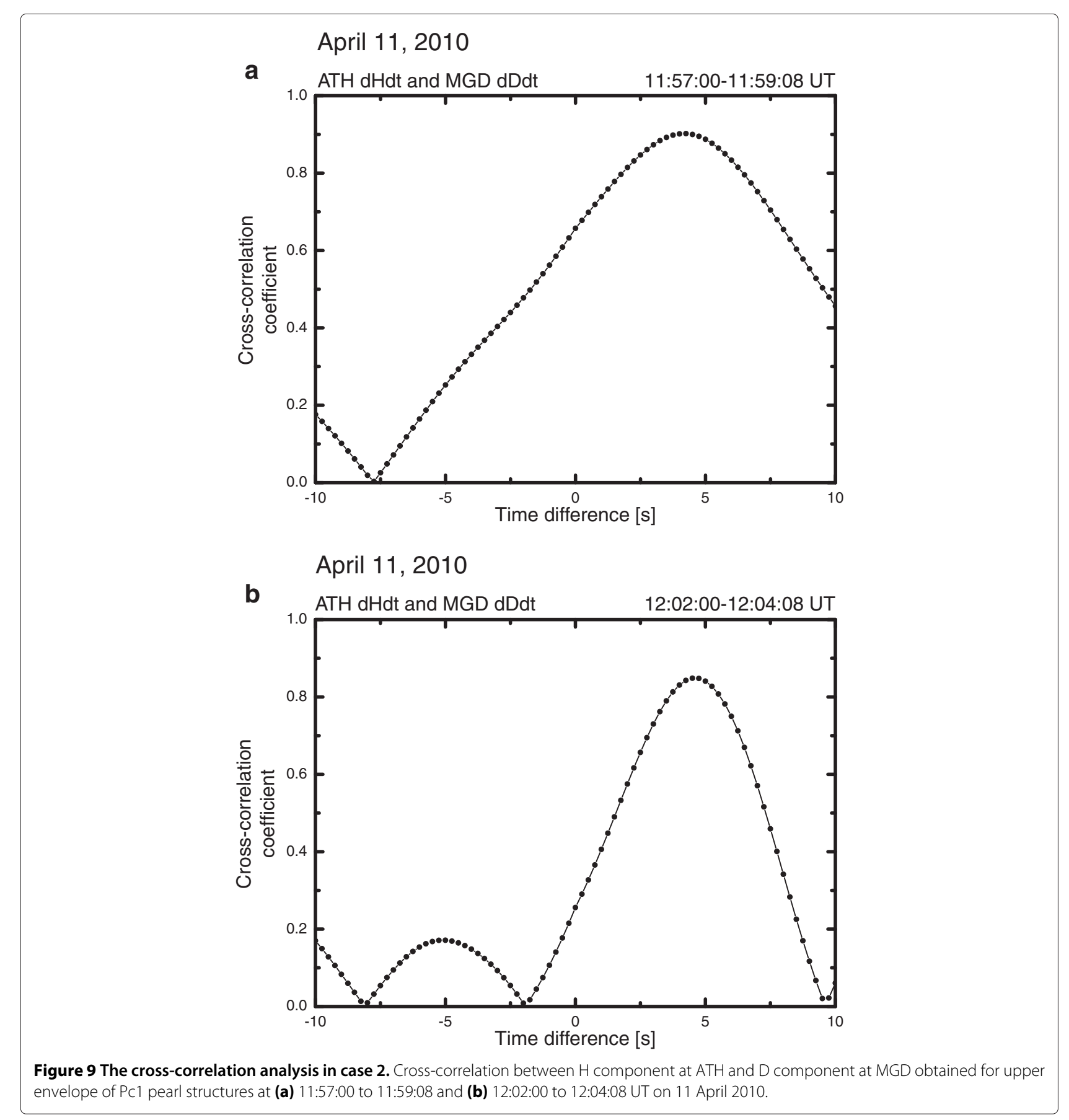

takes into account that Pc1 waves with different frequencies are mixed at a point source in the ionosphere (orange point in Figure 10b), corresponding to pearl structures created in the magnetosphere.

Figure 10a,b,c and Figure 10d,e,f show the results of models 1 and 2, respectively. For model 1, the source region is distributed from north to south with a length of 1,000 km. The waveforms of Pc1 waves in Figure 10b,c show that Pc1 pearl structures are slightly different at different stations, particularly for a station located at $90^{\circ}$ (black dot in Figure 10a and black lines in Figure 10b,c), corresponding to a perpendicular direction from the source distribution. Additionally, the time difference between two stations with the same distance from the source region varies because of the changing angle of the stations from the south. In the case of model 2, as shown in Figure 10d,e,f, Pc1 waves coming from a point ionospheric source have identical waveforms at different 

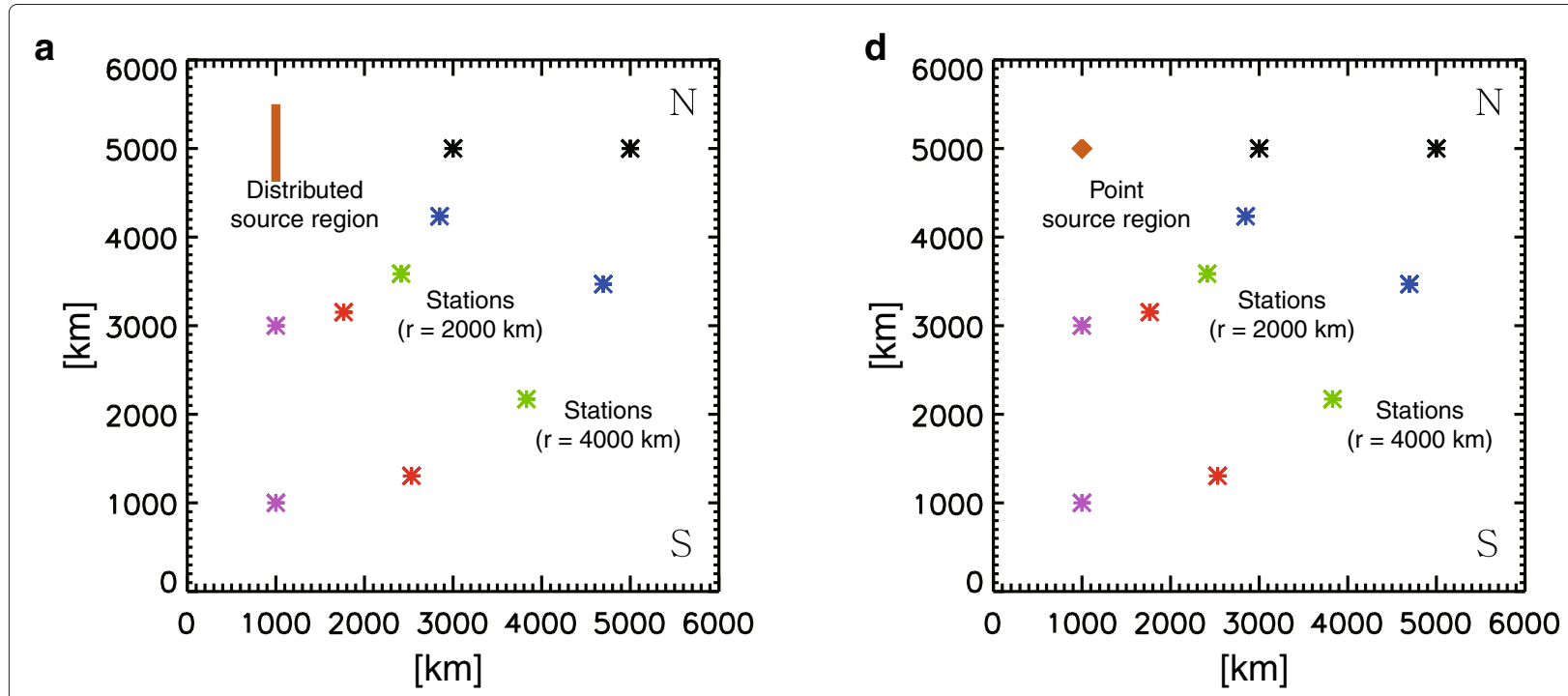

b

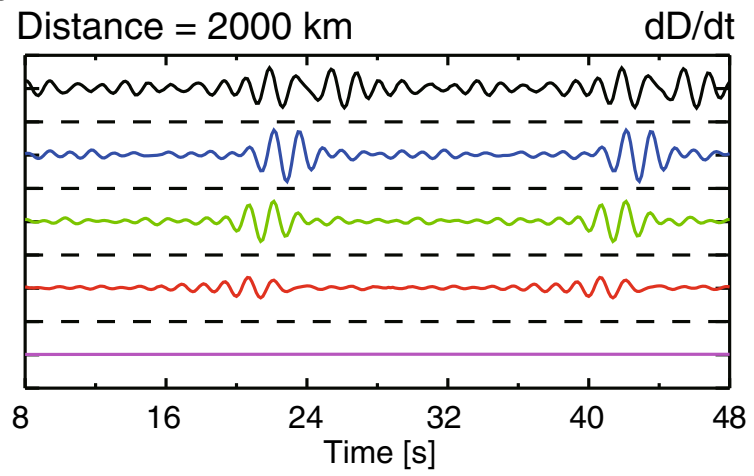

e

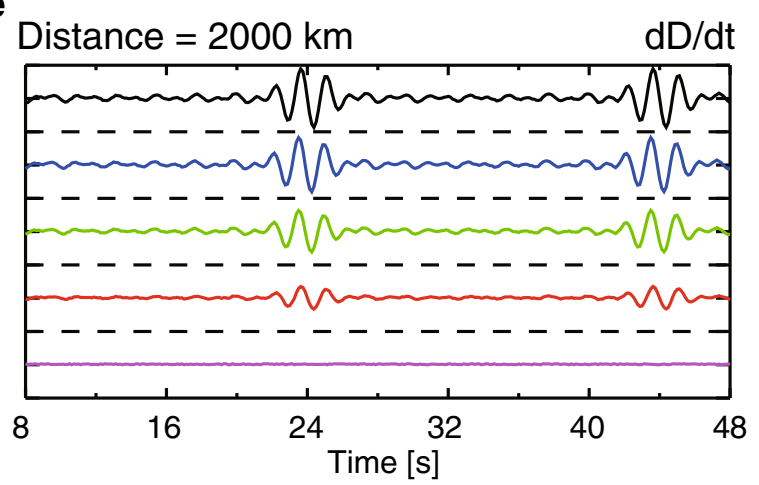

C

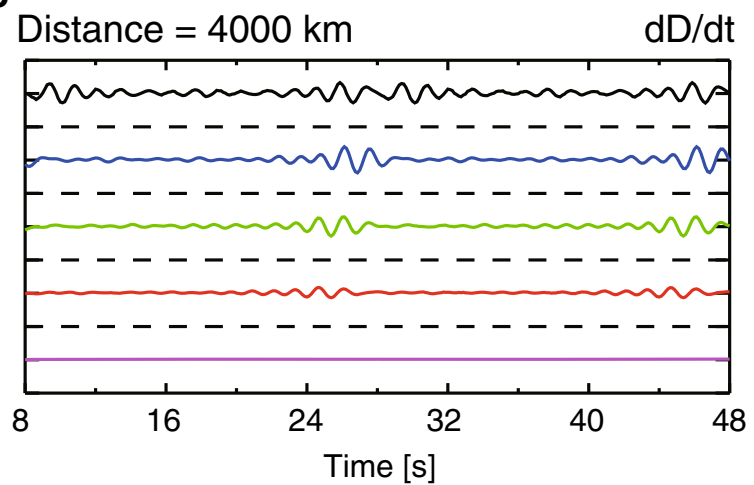

$\mathbf{f}$

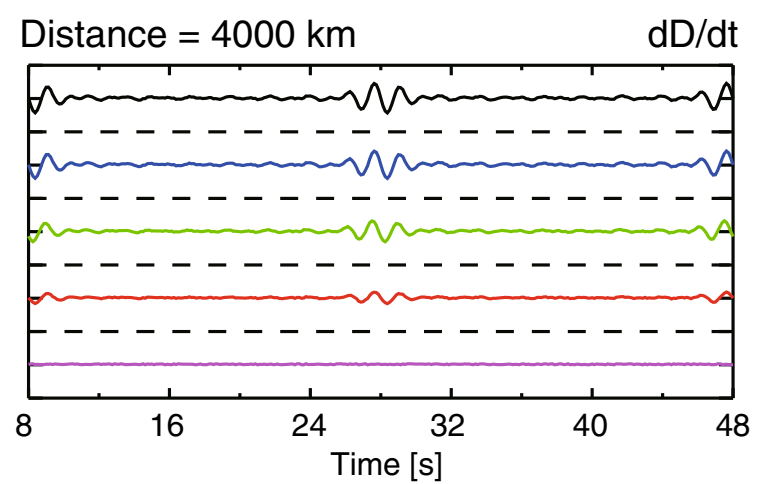

Figure 10 Simple model calculations for comparison between a distributed source and a point source. Location of stations and source region: (a) A distributed source region (model 1) and (d) a point source region (model 2). The D component waveforms of the source waves with frequencies of 0.6 to $1.0 \mathrm{~Hz}$ : distance from the source region to stations at (b) 2,000 and (c) 4,000 km for model 1 and (e) 2,000 and (f) 4,000 km for model 2. Colors indicate angles of stations from the south.

stations. The time differences between two different stations with the same distance from a source region are close to zero. If the Pc1 waves propagated from a spatially distributed source region in the ionosphere, the different Pc1 pearl structures would be observed at different stations due to beating processes in the ionosphere, even though the waves are coming from the same ionospheric source region. In case 1 , we found that Pc1 pearl structures were slightly different in some time intervals, with high coherence of Pc1 waveforms. We also found that the variation of 
polarization angle at the three stations depended on frequency. In case 2, however, the Pc1 pearl structures were similar, and the polarization angle was independent of frequency. As shown in the model calculations of Pc1 waves, we suggest that the observed case 1 could be caused by beating processes in the ionosphere, while Pc1 pearl structures in case 2 could be created by magnetospheric effects.

In addition, we cannot exclude the possible effects that dispersive propagation could have on ducted Pc1 waves. The effect can also contribute to the formation of Pc1 pearl structures in the ionosphere. Because the group velocity of dispersive waves differs from the phase speed, it can cause the modulation of wave amplitude in a wave packet. The high-latitude transmission and reflection properties of the ionosphere in the Pc1 frequency range is related to the wave number $\omega$ and the wave vector $\kappa$ (Greifinger 1972). As shown by model calculations by Fujita $(1987,1988$, the group velocity of Pc1 pulsations as a function of frequency increases near the lower cut off frequency. If the observed Pc1 waves have a broad bandwidth, the amplitude modulation of Pc1 waves could be caused by dispersive propagation through the ionospheric duct. From our observations, Pc1 pearl structures can have different shapes at different stations (case 1) and similar shapes at different stations (case 2). The bandwidth of case 1 (approximately $0.5 \mathrm{~Hz}$ ) was wider than that of case 2 approximately, suggesting that dispersive propagation contributes more to the creation of Pc1 pearl structures in the first case. However, in this study, we cannot quantify the contribution of this effect to the creation of Pc1 pearl structures in the ionosphere.

\section{Conclusions}

From 1 January 2009 to 31 December 2011, we investigated pearl structures of Pc1 geomagnetic pulsations observed by induction magnetometers at three mid- to low-latitude ground stations (ATH, MGD, and MOS). We selected two Pc1 pulsation events observed simultaneously at three stations: case 1 on 8 April 2010 and case 2 on 11 April 2010. The results of this study can be summarized as follows:

1. For case 1, even though the coherence of Pc1 waveforms at different stations is high, the Pc1 pearl structures were slightly different at different stations in some time intervals. The polarization angle varied depending on frequency, indicating that the Pc1 pulsations propagated from a spatially distributed ionospheric source.

2. For case 2, the Pc1 pearl structures are similar at different stations with high coherence of Pc1 waveforms. The polarization angle was almost constant, indicating that the source region of Pc1 pulsation is positioned in a localized region in the high-latitude ionosphere.

3. Pc1 pearl structures with a repetition period of approximately $10 \mathrm{~s}$ in case 1 and approximately 10 to approximately $40 \mathrm{~s}$ in case 2 were observed at three stations. These periods are shorter than those expected based on the BWP model.

4. From the model calculation of Pc1 pearl structures, we found that the pearl structures propagating from an ionospheric point source should have identical waveforms at different stations. The pearl structures generated by beating in the ionosphere with a spatially distributed source can be different at different stations.

From these results, we suggest that beating processes in the ionosphere with a spatially distributed ionospheric source can cause pearl structures during the ionospheric duct propagation from high to low latitudes, with long distances from the source to the stations. In case 2, however, we cannot reliably interpret the Pc1 pearl structures with a constant polarization angle using the beating process in the ionosphere. Therefore, we cannot exclude the possibility that mechanisms in the magnetosphere also contribute to the generation of Pc1 pearl structures. In order to understand and quantify the contribution of beating in the ionosphere to the creation of Pc1 pearl structures, we would like to further investigate the statistical characteristics in future studies.

\section{Competing interests}

The authors declare that they have no competing interests.

\section{Authors' contributions}

CWJ carried out the spectral analysis for case 1 and case 2, prepared simple model calculations for comparison between a distributed source and a point source, and wrote the manuscript. KS helped in planning for the design of the study and interpretation and drafted the manuscript as the supervisor of CWJ. $\mathrm{MC}$ and IS carried out the induction magnetometer observation at ATH in Canada and drafted the manuscript. IP and BS carried out the induction magnetometer observation at MGD in Russia and drafted the manuscript. All authors read and approved the final manuscript.

\section{Acknowledgements}

We thank M. Sera and Y. Ikegami at the Moshiri observatory of the Solar-Terrestrial Environment Laboratory, Nagoya University, all the staff of the Institute of Cosmophysical Research and Radiowave Propagation (IKIR), and Y. Katoh, H. Hamaguchi, and Y. Yamamoto of STEL, Nagoya University, for their help and support in the operation of the induction magnetometers. The Dst and $A E$ indices were provided by the WDC-C2 for geomagnetism at Kyoto University. This work was supported by Grants-in-Aid for Scientific Research (16403007, 18403011, 19403010, and 20244080), the 21th Century COE Program (Dynamics of the Sun-Earth-Life Interactive System, No. G-4), the Global COE Program of Nagoya University 'Quest for Fundamental Principles in the Universe (QFPU)', the Special Funds for Education and Research (Energy Transport Processes in Geospace), and the IUGONET Project from MEXT, Japan, as well as the Leadership Development Program for Space Exploration and Research from Nagoya University for Leading Graduate Schools.

\section{Author details}

${ }^{1}$ Solar-Terrestrial Environment Laboratory, Nagoya University, Furo-cho, Chikusa-ku, Nagoya 464-8601, Japan. ${ }^{2}$ Center for Science, Athabasca 
University, 1 University Drive, Athabasca AB T9S 3A3, Canada. ${ }^{3}$ Institute of Cosmophysical Research and Radiowave Propagation, Far Eastern Branch of the Russian Academy of Sciences, 7 Mirnaya Street, Paratunka, Kamchatka region, Russian Federation, 684034

Received: 28 March 2014 Accepted: 1 October 2014

Published online: 23 October 2014

\section{References}

Anderson BJ, Erlandson RE, Zanetti LJ (1992) A statistical study of PC 1-2 magnetic pulsations in the equatorial magnetosphere: 1. equatoria occurrence distributions. J Geophys Res Space Phys 97:3075-3088

Campbell WH (1967) Low attenuation of hydromagnetic waves in the ionosphere and implied characteristics in the magnetosphere for Pc 1 events. J Geophys Res 72(13):3429-3445

Erlandson R, Zanetti L, Potemra T, Block L, Holmgren G (1990) Viking magnetic and electric field observations of Pc 1 waves at high latitudes. J Geophys Res Space Phys 95(A5):5941-5955

Fowler R, Kotick B, Elliott R (1967) Polarization analysis of natural and artificially induced geomagnetic micropulsations. J Geophys Res 72(11):2871-2883

Fraser B (1975) lonospheric duct propagation and PC 1 pulsation sources. J Geophys Res 80(19):2790-2796

Fujita S (1987) Duct propagation of a short-period hydromagnetic wave based on the international reference ionosphere model. Planet Space Sci 35(1):91-103

Fujita S (1988) Duct propagation of hydromagnetic waves in the upper ionosphere, 2, dispersion characteristics and loss mechanism. J Geophys Res Space Phys 93(A12):14674-14682

Fukunishi H, Toya T, Koike K, Kuwashima M, Kawamura M (1981) Classification of hydromagnetic emissions based on frequency-time spectra. J Geophys Res Space Phys 86(A11):9029-9039

Greifinger P (1972) lonospheric propagation of oblique hydromagnetic plane waves at micropulsation frequencies. J Geophys Res 77(13):2377-2391

Guglielmi A, Feygin F, Mursula K, Kangas J, Pikkarainen T, Kalisher A (1996) Fluctuations of the repetition period of Pc1 pearl pulsations. Geophys Res Lett 23(9):1041-1044

Jacobs J, Watanabe T (1964) Micropulsation whistlers. J Atmos Terr Phys 26(8):825-826

Kawamura M, Kuwashima M, Toya T (1981) Comparative study of magnetic Pc1 pulsations between low latitudes and high latitudes: source region and propagation mechanism of the waves deduced from the characteristics of the pulsations at middle and low latitudes. Mem Natl Inst Polar Res. Special issue 18:83-100

Kim H, Lessard M, Engebretson M, Young M (2011) Statistical study of Pc1-2 wave propagation characteristics in the high-latitude ionospheric waveguide. J Geophys Res Space Phys 116(A7):07227

Kuwashima M, Toya T, Kawamura M, Hirasawa T, Fukunishi H, Ayukawa M (1981) Comparative study of magnetic Pc1 pulsations between low latitudes and high latitudes: statistical study. Mem Natl Inst Polar Res. Special issue 18:101-117

Manchester R (1966) Propagation of Pc 1 micropulsations from high to low latitudes. J Geophys Res 71(15):3749-3754

Mursula K (2007) Satellite observations of Pc 1 pearl waves: the changing paradigm. J Atmos Sol Terr Phys 69(14):1623-1634

Mursula K, Rasinkangas R, Bösinger T, Erlandson R, Lindqvist P-A (1997) Nonbouncing PC 1 wave bursts. J Geophys Res Space Phys 102(A8):17611-17624

Mursula K, Kangas J, Kerttula R, Pikkarainen T, Guglielmi A, Pokhotelov O, Potapov A (1999) New constraints on theories of PC1 pearl formation. J Geophys Res Space Phys 104(A6):12399-12406

Mursula K, Bräysy T, Niskala K, Russell C (2001) Pc1 pearls revisited: structured electromagnetic ion cyclotron waves on polar satellite and on ground. J Geophys Res Space Phys 106(A12):29543-29553

Nomura R, Shiokawa K, Pilipenko V, Shevtsov B (2011) Frequency-dependent polarization characteristics of $\mathrm{PCl}$ geomagnetic pulsations observed by multipoint ground stations at low latitudes. J Geophys Res Space Phys 116(A1):01204

Nomura R, Shiokawa K, Sakaguchi K, Otsuka Y, Connors M (2012) Polarization of $\mathrm{pc} 1 / \mathrm{EMIC}$ waves and P related proton auroras observed at subauroral latitudes. J Geophys Res Space Phys 117(A2):02318

Obayashi T (1965) Hydromagnetic whistlers. J Geophys Res 70:1069-1078
Paulson K, Smith C, Lessard M, Engebretson M, Torbert R, Kletzing C (2014) In situ observations of Pc1 pearl pulsations by the van allen probes. Geophys Res Lett 41(6):1823-1829

Perraut S (1982) Wave-particle interactions in the ulf range: geos-1 and-2 results. Planet Space Sci 30(12):1219-1227

Pope JH (1964) An explanation for the apparent polarization of some geomagnetic micropulsations (pearls). J Geophys Res 69(3):399-405

Rasinkangas R, Mursula K (1998) Modulation of magnetospheric emic waves by Pc 3 pulsations of upstream origin. Geophys Res Lett 25(6):869-872

Sakaguchi K, Shiokawa K, Miyoshi Y, Otsuka Y, Ogawa T, Asamura K, Connors M (2008) Simultaneous appearance of isolated auroral arcs and Pc 1 geomagnetic pulsations at subauroral latitudes. J Geophys Res Space Phys 113(A5):05201

Shiokawa K, Nomura R, Sakaguchi K, Otsuka Y, Hamaguchi Y, Satoh M, Katoh Y, Yamamoto Y, Shevtsov B, Smirnov S, Poddelsky I, Connors M (2010) The STEL induction magnetometer network for observation of high-frequency geomagnetic pulsations. Earth Planets Space 62(6):517

Tepley L, Landshoff R (1966) Waveguide theory for ionospheric propagation of hydromagnetic emissions. J Geophys Res 71(5):1499-1504

Troitskaya V, Gul'Elmi A (1967) Geomagnetic micropulsations and diagnostics of the magnetosphere. Space Sci Rev 7(5-6):689-768

Usanova M, Mann I, Rae I, Kale Z, Angelopoulos V, Bonnell J, Glassmeier K-H, Auster $\mathrm{H}$, Singer $\mathrm{H}$ (2008) Multipoint observations of magnetospheric compression-related EMIC PC1 waves by themis and carisma. Geophys Res Lett 35(17):17-25

Waters C, Lysak R, Sciffer M (2013) On the coupling of fast and shear Alfvén wave modes by the ionospheric hall conductance. Earth Planets Space 65(5):385-396

doi:10.1186/s40623-014-0140-8

Cite this article as: Jun et al:: Study of Pc1 pearl structures observed at multi-point ground stations in Russia, Japan, and Canada. Earth, Planets and Space 2014 66:140.

\section{Submit your manuscript to a SpringerOpen ${ }^{\circ}$ journal and benefit from:}

- Convenient online submission

- Rigorous peer review

- Immediate publication on acceptance

- Open access: articles freely available online

- High visibility within the field

- Retaining the copyright to your article

Submit your next manuscript at $>$ springeropen.com 